\title{
THE SLEWING MIRROR TELESCOPE AND THE DATA-ACQUISITION SYSTEM FOR THE UFFO-PATHFINDER
}

H. Lim ${ }^{1}$, S. Ahmad ${ }^{2}$, P. Barrillon ${ }^{2}$, S. Brandt ${ }^{3}$, C. Budtz-Jørgensen ${ }^{3}$, A.J. Castro-Tirado ${ }^{4}$, P. Chen ${ }^{5}$, Y.J. Choi ${ }^{6}$, P. Connell ${ }^{7}$, S. Dagoret-Campagne ${ }^{2}$, C. Eyles ${ }^{7}$, B. Grossan ${ }^{8}$, M.-H.A. Huang ${ }^{9}$, A. Jung ${ }^{10}$, S. Jeong ${ }^{10}$, J.E. Kim ${ }^{10}$, M.B. Kim ${ }^{1}$, S.-W. Kim ${ }^{11}$, Y.W. Kim ${ }^{1}$, A.S. Krasnov ${ }^{12}$, J. Lee ${ }^{1}$, E.V. Linder ${ }^{8,10}$, T.-C. Liu ${ }^{5}$, N. Lund ${ }^{3}$, K.W. $\mathrm{Min}^{6}$, G.W. Na ${ }^{10}$, J.W. Nam ${ }^{5}$, M.I. Panasyuk ${ }^{12}$, I.H. Park ${ }^{1}$, J. Ripa ${ }^{1}$, V. Reglero ${ }^{7}$, J.M. Rodrigo ${ }^{7}$, G.F. Smoot ${ }^{8,10}$, J.E. Suh ${ }^{10}$, S. Svertilov ${ }^{12}$, N. Vedenkin ${ }^{12}$, M.-Z. Wang ${ }^{5}$ and I. Yashin ${ }^{12}$

\begin{abstract}
The Ultra-Fast Flash Observatory (UFFO) aims to detect the earliest moment of Gamma-Ray Bursts (GRBs) which is not well known, resulting into the enhancement of GRB mechanism understanding. The pathfinder mission was proposed to be a scaled-down version of UFFO, and only contains the UFFO Burst Alert \& Trigger Telescope (UBAT) measuring the X-ray/gamma-ray with the wide-field of view and the Slewing Mirror Telescope (SMT) with a rapid-response for the UV/optical photons. Once the UBAT detects a GRB candidate with the position accuracy of $10 \mathrm{arcmin}$, the SMT steers the UV/optical photons from the candidate to the telescope by the fast rotatable mirror and provides the early UV/optical photons measurements with 4 arcsec
\end{abstract}

\footnotetext{
1 Sungkyunkwan University, Suwon, Korea

2 University of Paris-Sud 11, Orsay, France

3 Technical University of Denmark, Copenhagen, Denmark

${ }^{4}$ Instituto de Astrofísica de Andalucía, CSIC, Granada, Spain

5 National Taiwan University, Taipei, Taiwan

${ }^{6}$ Korea Advanced Institute of Science and Technology, Daejeon, Korea

7 University of Valencia, Valencia, Spain

${ }^{8}$ University of California, Berkeley, USA

9 National United University, Miao-Li, Taiwan

10 Ewha Womans University, Seoul, Korea

11 Yonsei University, Seoul, Korea

12 Moscow State University, Moscow, Russia
} 
accuracy. The SMT has a modified Ritchey-Chrètien telescope with the aperture size of $10 \mathrm{~cm}$ diameter including the rotatable mirror and the image readout by the intensified charge-coupled device. There is a key board called the UFFO Data Acquisition system (UDAQ) that manages the communication of each telescope and also of the satellite and the UFFO overall operation. This pathfinder is designed and built within the limited size and weight of $\sim 20 \mathrm{~kg}$ and the low power consumption up to $\sim 30 \mathrm{~W}$. We will discuss the design and performance of the UFFO-pathfinder, and its integration to the Lomonosov satellite.

\section{Introduction}

Gamma-Ray Bursts (GRBs) are the most energetic events in the sky with the higher photon luminosities than any other objects in the universe and seen to the highest red-shift. They have been detected in random directions in the sky once or twice per day and have lasted from a fraction of seconds to hundred seconds.

Compared with X-ray observations of GRBs, the optical photons are observed in $40 \%$ of X-ray events. The Swift observatory detecting the multi-wavelength photons of GRBs and the ground-based fast robotic telescope rarely have observed the UV/optical photons in less than $60 \mathrm{sec}$ after the GRB trigger. The UltraFast Flash Observatory (UFFO) was proposed to detect the earliest explosion moments associated with GRBs and to study the GRBs emission mechanism, their progenitors etc.. The UFFO is designed to begin the UV/optical observation in less than a few seconds after trigger with the beam steering technology which is implemented in the Slewing Mirror Telescope (SMT). The UFFO-pathfinder, a pilot GRB mission is scheduled to launch into orbit in 2013 by the Lomonosov spacecraft to verify the early UV/optical measurements of GRBs with the fast rotatable mirror system. There are two telescopes and one data acquisition system: the UFFO Burs Alert \& Trigger Telescope (UBAT) for the X-ray triggering, the SMT for the UV/optical measurement and the UFFO Data Acquisition System (UDAQ).

The UBAT is a coded-mask aperture X-ray camera with a wide field of view (FOV) of $1.8 \mathrm{sr}$ and the detector module with effective active area of $191.1 \mathrm{~cm}^{2}$, localizing the GRBs within the accuracy of 10 arcmin diameter. The SMT slews the motorized mirror rapidly forward to the target triggered by UBAT within a second and measures UV/optical afterglow with a Ritchey-Chrètien telescope and a focal plane detector. The UDAQ controls the operation and communication of each telescope and also interfaces with the satellite. As shown in Figure 1 (left), the UFFO-pathfinder was designed and built with the constraints of the mass of $20 \mathrm{~kg}$ and the maximum length of $800 \mathrm{~mm}$ required by the spacecraft. The logic functions of UFFO's each readout system were implemented in the field programmable gates arrays (FPGAs) and are operated with the low power consumption and the fast signal processing.

In this paper, we will discuss the design, fabrication and performance test of the SMT and the UDAQ that are integrated in the UFFO-pathfinder. 


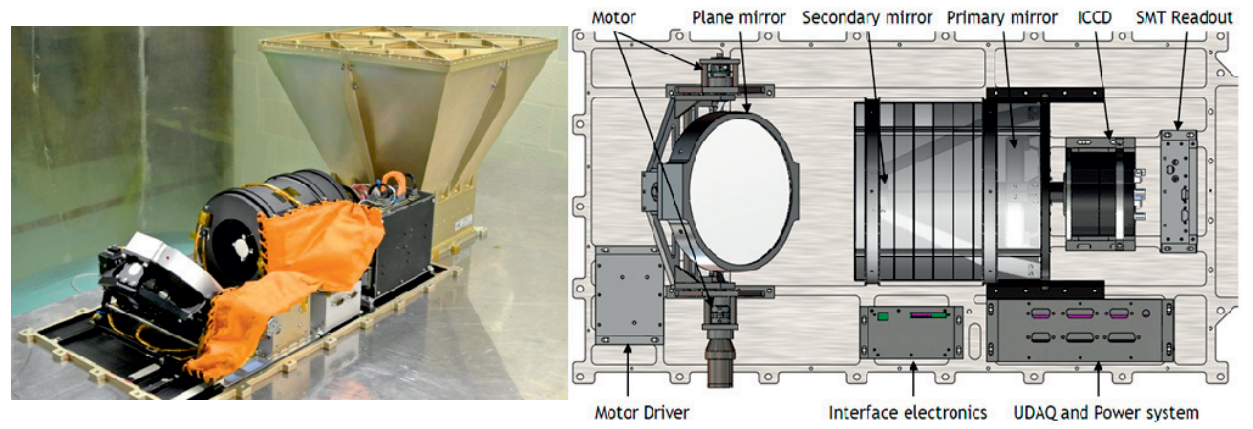

Fig. 1. Left: picture of UFFO-pathfinder without the SMT case. Right: schematic view of the SMT assembly.

\section{Slewing mirror telescope}

The SMT provides the fast pointing to the target with the narrow FOV using a fast steerable mirror plate. It is a Ritchey-Chrètien telescope with a $100 \mathrm{~mm}$ diameter aperture using a motorized mirror plate and with an f-number of 11.4 . The FOV of SMT is $17 \times 17 \mathrm{arcmin}^{2}$ and each pixel corresponds to the angular size of $4 \times 4 \operatorname{arcsec}^{2}$. The wavelength coverage is $200 \sim 650 \mathrm{~nm}$. The overall size is $622.5(\mathrm{~L}) \times 400(\mathrm{~W}) \times 210(\mathrm{H}) \mathrm{mm}^{3}$. With the mass of $11.5 \mathrm{~kg}$ and the power of $10 \mathrm{~W}$, the optics, the mechanical structure and the electronics were designed and fabricated. When the UBAT finds the GRB candidates and sends their location information to the SMT via the UDAQ interface, the SMT calculates the motor slewing angle, tilts the rotatable mirror to UBAT's detected target as following the angle and takes its UV/Optical data. This process is done within seconds after a GRB is triggered and consequently the UFFO can detect the early emission from GRBs.

\subsection{Ritchey-Chrétien telescope}

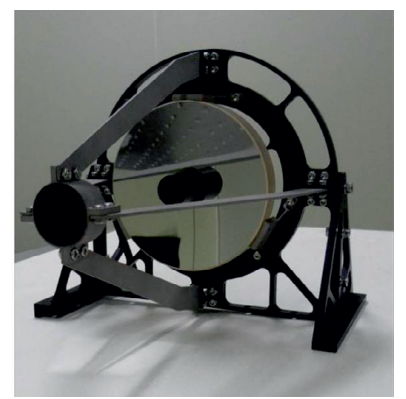

Fig. 2. Photograph of the assembled Ritchey-Chrétien Telescope with $10 \mathrm{~cm}$ of aperture. 
As shown in Figure 2, this telescope consists of a primary mirror of $100 \mathrm{~mm}$ in diameter and -1.01 in conic coefficient and a secondary mirror of $20 \mathrm{~mm}$ in diameter and -1.83 in conic coefficient, located about $130 \mathrm{~mm}$ away. It has the good imaging performance in Modulation Transfer Function (MTF) of 0.77 at the Nyquist frequency of $22.52 \mathrm{~mm}^{-1}$ and an on-axis RMS spot radius of $2.48 \mu \mathrm{m}$. The optical system is designed for sufficient stiffness within the tight mass budget and for the proper optical performance with the thermal stability. The obscuration ratio of the assembled telescope is $12.5 \%$.

The optical alignment of SMT telescope was measured using an interferometer. With respect to the primary mirror, the secondary mirror was aligned by adjusting five movements of $\mathrm{X} \& \mathrm{Y}$ decenters, $\mathrm{X} \& \mathrm{Y}$ tilts and $\mathrm{Z}$ displacement. In order to satisfy with the required optics resolution, the RMS value of Wavefront Error (WFE) from the aligned optics should be less than 0.25 of the injected light source's wavelength. The measured error is less than $\lambda / 20$ using He-Ne $(632.8 \mathrm{~nm})$ laser and shows the good alignment and integration of SMT optics [1].

\subsection{Slewing mirror stage}
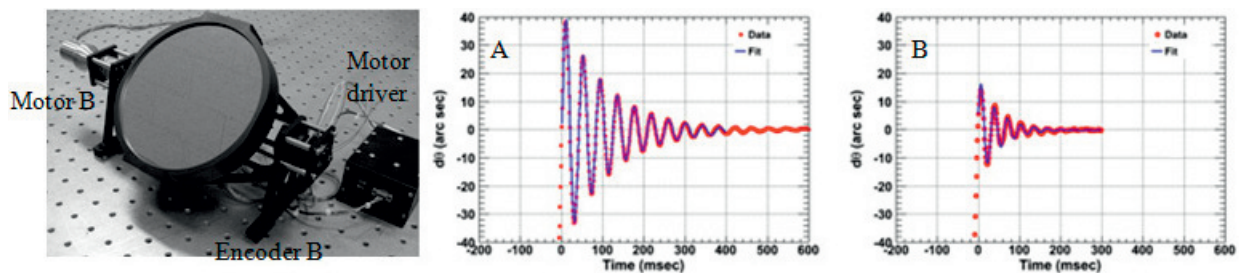

Fig. 3. Slewing mirror stage was shown (left). After slewing the mirror, the settling time measured less than $300 \mathrm{~ms}$ at A-axis (center) and at B-axis (right).

A slewing mirror (Fig. 3 (left)) with two-axis gimbal stage is located in front of a Ritchey-Chrétien telescope and points the target with the pointing accuracy of 1 arcmin. This tilting range is $\pm 35 \mathrm{deg}$ in sky coverage which corresponds into the half-coded FOV of UBAT. The mirror is driven by the stepping motors and a harmonic drive gear with 100:1 reduction ratio, providing the minimum step size of 4.05 arcsec. After slewing the mirror, the settling time for two-axis motors was measured to be less than $300 \mathrm{~ms}$ (Fig. 3 (center) and Fig. 3 (right)). In order to cover the $10 \mathrm{~cm}$ diameter of SMT optics, the Zerodur mirror with $15 \mathrm{~cm}$ diameter was used and weighted to be $482 \mathrm{~g}$ with the light-weighting factor of $57 \%$ [1].

\subsection{Focal plane detector and readout system}

For the focal plane detector, an Intensified Charge-Coupled Device (ICCD) was chosen and consists of a photocathode, two Micro-Channel Plates (MCPs) for photoelectron multiplication, a phosphor screen, a tapered fiber-optics and a CCD readout system with the detectable pixels of $256 \times 256$. For the customized ICCD, 
an UV-enhanced S20 material for the photocathode is used to detect the photons of $200-650 \mathrm{~nm}$. The photoelectrons from the photocathode are amplified by the gain of $10^{4} \sim 10^{6}$ in MCPs stage. They are converted to photons in the phosphor screen. This screen uses the P46 material with the fast decay time of $300 \mathrm{~ns}$ and its output photons are readout by a fast interline CCD, Kodak KAI-0340 which provides a fast pixel-readout rate up to $40 \mathrm{MHz}$. The phosphor screen is coupled with the CCD by the tapered fiber-optics with the ratio of $3.2: 1 \pm 3 \%$. Therefore, the actual focal plane size of SMT is $6.062 \times 6.062 \mathrm{~mm}^{2}$ and a pixel size is $23.7 \times 23.7 \mu \mathrm{m}^{2}$.

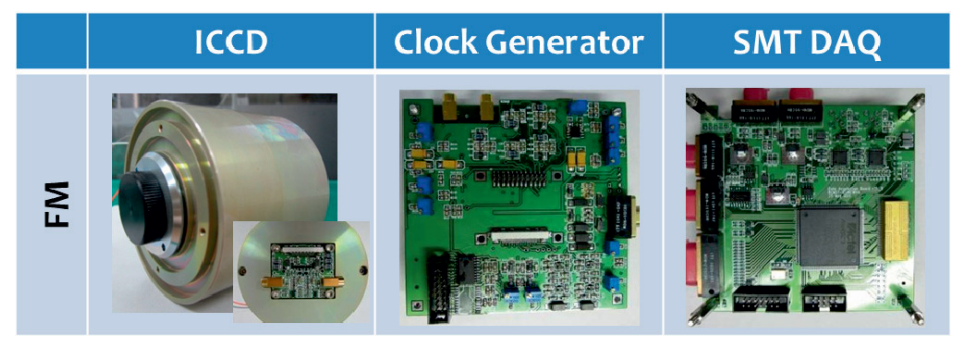

Fig. 4. ICCD including the CCD-sensor board (left), the clok-generator board (center) and the SMT-DAQ board (right).

In order to build the SMT readout system within the limited space and power, the readout system consists of three parts; the ICCD including the CCD-sensor board for sensor readout, the clock-generator board for the operation of CCD sensor, and the SMT-DAQ board for overall SMT operations including motor control and interface with UDAQ [2]. The CCD output is digitized with 10bit ADC and the readout rate of one frame is $4 \mathrm{~ms}$. When checking the integrated SMT using the parallel beam, we achieved the Point Spread Function of 4.8 arcsec.

\subsection{Data-acquisition system}

The UDAQ manages the overall operation of UFFO-pathfinder, and the interfaces with each telescope and also the satellite. The architecture of UFFO-pathfinder is shown schematically with the trigger flows, the data flows and the control flows in Figure 5. When the Satellite provides the power for UFFO-pathfinder, the UFFO power system produces the required voltages and first supports the voltages for UDAQ. The UDAQ automatically starts to run, controls to distribute the powers to SMT and UBAT and monitors the operation status and the hardware status, such as housekeeping data. All logic functions performed by the UDAQ are implemented in a field programmable gate arrays (FPGA) without a microprocessor. It supports the fast processing and the suitable operation for the sub-minute data observation [3]. Figure 6 shows the fabricated UDAQ board and the UFFO bus-interface board with the satellite. 
UFFO Control/Data/Trigger Architecture

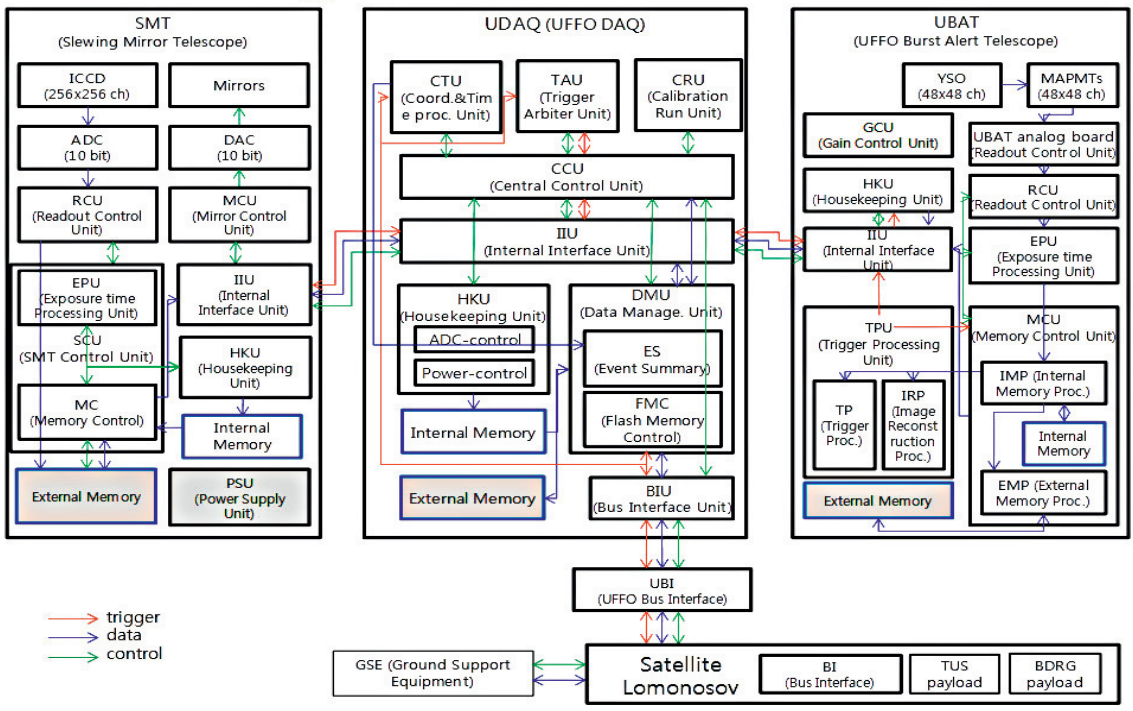

Fig. 5. Architecture of the UFFO-pathfinder including SMT, UDAQ, UBAT and satellite intefrace. There are the trigger flows (red line), the data flows (blue line), and the control flows (green line).

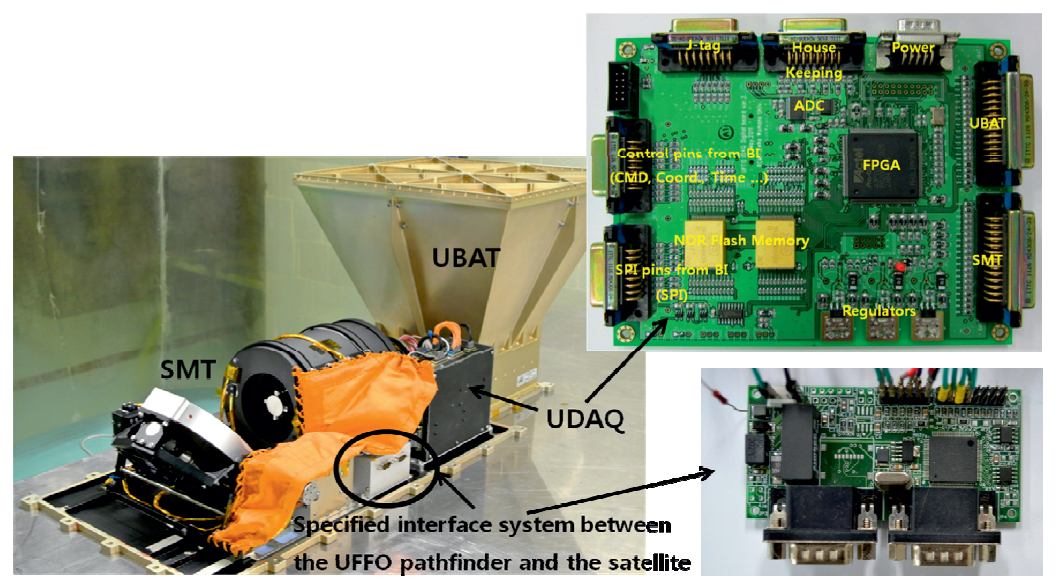

Fig. 6. UDAQ board (top-right) and the UFFO bus-interface board (bottom-right). The left picture shows the positions of these boards.

\section{Conclusion}

The Slewing Mirror Stage is a key instrument of UFFO and allows for us to detect the early UV/optical photons of GRBs. The Slewing Mirror Telescope 
which consists of the telescope, the slewing mirror stage and the readout system, is designed, built and integrated in the UFFO-pathfinder. The integrated SMT system is successfully delivered to Russia. The UFFO data-acquisition System is the main control system for UFFO-pathfinder and has been tested with each telescope and the satellite.

This research was supported by Basic Science Research Program through the National Research Foundation of Korea (NRF) funded by the Ministry of Education, Science and Technology (20100025056), Creative Research Initiatives (RCMST) of MEST/KRF, and supported by World Class University (WCU) program through the National Research Foundation of Korea funded by the Ministry of Education, Science and Technology (R32-2009-000-10130-0) in Korea.

\section{References}

[1] Jeong, S., Ahmad, S., Barrillon, P., et al., 2012, Proc. SPIE, 8443, 84432S

[2] Kim, J.E., Lim, H., Jung, A., et al., 2011, Proceeding of 2011 ICRC [arXiv: 1106.3803]

[3] Na, G.W., Ahmad, S., Barrillon, P., et al., 2012, Proc. SPIE, 8443, 84432 T 
